leaves in Illinois as influenced by soil type and soil composition. Soil Sci. 68: 317-328.

Metz, L. J., G. G. Wells, and B. F. Swindel. 1966. Sampling soil and foliage in a pine plantation. Soil Sci. Soc. Amer. Proc. 30: 397-399.

Moore, C. W. E. 1959. The interaction of species and soil in relation to the distribution of eucalypts. Ecology 40: 734-735.

- 1961. Competition between Eucalyptus melliodora and Eucalyptus rossii at varying levels of exchangeable calcium. Aust. J. Bot. 9: 92-97.

Mulder, D. 1953. Nutritional studies on fruit trees. II. The relation between potassium, magnesium and phosphorus in apple trees. Plant and Soil 4: 107117.

Pirson, A. 1956. Functional aspects in mineral nutrition of green plants. Ann. Rev. Plant Physiol. 6: 71-114.

Pryor, L. D. 1959 . Species distribution and association in Eucalyptus. p. 461-471. In A. Keast, R. L. Crocker and C. S. Christian (ed.) Biogeography and ecology in Australia. Dr. W. Junk. The Hague, Netherlands. 640 p.

Reuther, W., F. E. Gardner, P. F. Smith, and W. R. Roy. 1949. Phosphate fertilization trials with oranges in Florida. I. Effect on yield, growth, and leaf and soil composition. Amer. Soc. Hort. Sci. Proc. 53: 71-84.

Shomaker, C. E., and V. J. Rudolph. 1964. Nutritional relationships affecting height growth of planted yellow-poplar in southwestern Michigan. Forest Sci. 10: $66-76$.

Slatyer, R. P. 1967. Plant-water relationships. Academic Press, New York, 366 p.

Slatyer, R. P. and I. C. McIlroy. 1961. Practical microclimatology. CSIRO and UNESCO, Australia. 5.12-5.17.

Snedecor, G. W. 1956. Statistical methods. 5th ed. Iowa State Coll. Press, Ames, Iowa. 534 p.

Tanada, T. 1955. Effects of ultraviolet radiation and calcium and their interaction on salt absorption by excised Mung bean roots. Plant Physiol. 30: 221225.

Walker, L. C. 1956. Foliage symptoms as indicators of potassium-deficient soils. Forest Sci. 2: 113-210.

Walker, R. B. 1954. The ecology of serpentine soils. II. Ecology 35: 259-266.

Wiklander, L. 1958. The soil, p. 118-163. In W. Ruhland (ed.) Encycl. Pl. Physiol. IV, Springer Verlag. Berlin.

\title{
THE COMPETITIVE STRUCTURE OF COMMUNITIES : AN EXPERIMENTAL APPROACH WITH PROTOZOA
}

\author{
JoHN H. VANDERMEER ${ }^{1}$ \\ Department of Zoology, University of Michigan, Ann Arbor, Michigan
}

(Received November 25, 1968; accepted for publication January 31, 1969)

\begin{abstract}
An empirical test of the existence of higher order interactions was carried out using four ciliate protozoans, Paramecium caudatum, P. bursaria, P. aurelia, and Blepharisma sp.

All four ciliates were cultured individually, and their population histories were described quite well by the simple logistic equation.

Attempts to explain minor deviations of the data from the logistic by use of the one or two time lag logistic failed. A more complicated time lag phenomenon must be operative.

Every possible pair of the four ciliates was cultured and a trial and error procedure was used to estimate $\alpha$ and $\beta$ of the Gause equations. In all cases the simple Gause equations seemed adequately to describe the data.

All four ciliates were cultured together and compared to predictions made by use of the competition coefficients estimated from pair-wise competition and population parameters estimated from single species population growth. The correspondence between prediction and data suggests that the higher order interactions have slight or no effect on the dynamics of this artificial community.
\end{abstract}

The central goal of community ecology is to understand the dynamics of community organization. Most likely that goal would be best approached from the point of view of mechanism, beginning with basic principles and deducing how communities should behave. However, such attempts have in the past been stifled by certain methodological difficulties, diverting the attention of community ecologists toward more empirical observations. These empirical observations have usually taken the form of measuring the relative

${ }^{1}$ Present address: Department of Biology, University of Chicago, 1103 E. 57th Street, Chicago, Illinois 60637. abundance of species (number of species with a given number of individuals).

Most of the work concerned with the latter may be dichotomized as follows. One series of papers (Fisher, Corbet, and Williams 1943; Preston 1948, 1962a, b; Hairston and Beyers 1954; Hairston 1959) emphasizes underlying mathematical distributions and causes of deviance from these distributions. Another series, stimulated by Margalef (1957), and rigorously formalized by Pielou (1966a, b), is concerned with measuring the relative abundance of species, usually to compare communities to each other. 
There have also been some attempts at building either conceptual or mathematical models of communities using supposedly basic components. Kendall (1948) has shown how certain patterns of population growth will lead to Fisher's logarithmic series. MacArthur (1955) discussed several aspects of community stability as deduced from considerations of energy transfer among populations, and Watt (1964) later tested some of these ideas. MacArthur (1960) suggested mechanisms whereby lognormal distributions should arise. Hairston, Smith, and Slobodkin (1960) presented a general explanation for the structure of terrestrial communities which stimulated a recent controversy (Murdoch 1966, Ehrlich and Birch 1967, Slobodkin, Smith, and Hairston 1967). Garfinkel (1967) studied stability properties of theoretical systems using the Lotka-Volterra predator-prey equations.

These approaches to community ecology have established the basic attitude for the following empirical tests. Simple assumptions are made, namely that populations grow according to the logistic equation, and populations on the same trophic level obey the Gause equations of competition. I shall be concerned with communities which derive their structure through inter- and intraspecies competition.

The basic Gause competition equations may be extended to include $m$ species. The differential equation for the $i$ th species is,

$$
\frac{\mathrm{d} N_{i}}{d t}=\frac{r_{i} N_{i}}{K_{i}}\left\{K_{i}-N_{i}-\sum_{j=1}^{m} \alpha_{i j} N_{j}\right\} j \neq i
$$

(MacArthur and Levins 1967), where there are $m$ species in the community, $\alpha_{i j}$ is the effect of the $j$ th species on the $i$ th species, $r$ is the intrinsic rate of natural increase, $N$ is number of individuals and $K$ is saturation density. Assumed in the above extension is that there are no higher order interaction terms. That is, we need not write,

$$
\frac{\mathrm{d} N_{i}}{d t}=\frac{r_{i} N_{i}}{K_{i}}\left\{K_{i}-N_{i}-\Sigma \alpha_{i j} N_{j}-\sum_{j k} \beta_{i j k} N_{j} N_{k}-\ldots-\underset{j k \ldots m}{\Sigma} \omega_{i j k \ldots m} N_{j} N_{k} \ldots N_{m}\right\}
$$

where $j \neq i, k \neq i, \ldots, m \neq i$, and the Greek letters are interaction coefficients of increasingly higher order, e.g. $\beta_{i j k}$ is the joint effect of species $j$ and $k$ on the $i$ th species. Communities represented by equations (1) will be called noninteractive communities, and communities represented by equations (2) will be called interactive communities.

Equations (1) (noninteractive communities) are fairly tractable and have already led to some rather interesting conclusions about community structure (Levins 1968, Vandermeer 1968). On the other hand, equations (2) (interactive communities) are difficult to work with analytically.

The qualitative effects of higher order interactions are made clear in the following discussion. Consider the competitive effect, on one particular species (say species A), of adding further species, holding the average $\alpha$ constant and assuming the higher order terms to be negligible. The mean $\alpha$ is held constant in such a way that each new species overlaps with $25 \%$ of species A. If another species is added, species A only occupies $75 \%$ of its former niche without competitive pressure. If another species is added only $50 \%$ of its niche is free of competition. In general, as competitors accumulate in the system, if the average competition coefficient remains constant, the weight of competition eventually gets so strong that other species cannot make their way into the community.
Now suppose the three way interactions (the $\beta$ 's in equation (2)) are important. Considering again species $A$, after the addition of another species, as before only $75 \%$ of the former niche is occupied without competition. If an additional species is added, part of the niche overlap will be contained in a three way overlap. Thus, instead of having the niche reduced to $50 \%$ of its former size, something greater than $50 \%$ but less than $75 \%$ of the niche remains free of competition (since a portion of the potential competition is absorbed in the three way interaction).

For instance, consider a hypothetical organism living in a stream. Suppose specics A occupies the entire length of the stream. Suppose further that experiments are undertaken in which species $B$ and $C$ are added one at a time. If $B$ and $C$ each have $\mathrm{X}$ effect on species $\mathrm{A}$, we would expect that the combination of $\mathrm{B}$ and $\mathrm{C}$ together would have effect $2 X$. But suppose that the result of $B$ and $C$ competing with $A$ is that in either case $A$ is eliminated only from the upstream section of the stream. It does not matter to species $A$ which of $B$ or $C$ does the eliminating; the fact is, it is being eliminated. Thus, the predicted effect of $B$ and $C$ together on $A$ would be $2 \mathrm{X}$ but the observed effect would be $X$.

The type of higher order interaction discussed above implies $\beta, \gamma, \ldots, \omega$ of equation 4 are positive. If, on the other hand, the higher order terms were negative, it would imply a coalition formed 
by two or more species against some other species. The higher order interaction reported by Hairston, et al. (1969) is an example of a coalition type interaction.

Thus, it is not possible at the present time to predict the general effect of higher order interactions except qualitatively. However, it is clear that their effects may be highly significant. It, therefore, would be quite important to discover whether or not they commonly occur in nature.

The motivation for undertaking the experiments reported below derives from the foregoing discussion of interactive communities. Since several interesting consequences are deducible from the premises of noninteractive communities, it would be desirable to know either the importance of higher order interactions in real situations, or the theoretical effect which higher order interactions would have on the consequences derived from the noninteractive assumptions. As remarked earlier, it is virtually certain that higher order interactions will have an effect on the outcomes of the model, but the nature and extent of this effect are difficult to assess owing to the intractable nature of the model when applied to interactive communities. Thus, the simplest approach is to verify the presence or absence of higher order interactions in nature, and this is the approach of this paper.

Induction in biology - the implied approach suggested in the above discussion-has often been eased by first studying extremes. If the existence of higher order interactions can be demonstrated for a group of species which would be expected on an a priori basis not to exhibit them, it may be safely assumed that such interactions are more or less of universal occurrence. If that group of species does not exhibit higher order interactions, the broad implications for which the experiment was designed are lost.

Obtaining an extreme example and satisfying the basic postulates of the model have been the two guidelines in choosing experimental subjects and situations. Different species of free-living ciliates from different localities seem to satisfy both of these guidelines. Ciliates are simple organisms from the population point of view, and in several cases are known to conform to simple population mathematics, making it likely that most of the basic premises of the model are satisfied.

Therefore, four species of ciliates from four different localities were studied in a laboratory situation. Parameters of population growth were estimated for each species separately. Competition coefficients were estimated for each possible pairwise combination of species. Then all four species were cultured together and compared to the prediction made on the basis of the simple growth parameters and competition coefficients measured earlier.

\section{Methods and Materials}

Three of the four ciliates, Paramecium bursaria (PB), Paramecium aurelia (PA), and Paramecium caudatum (PC) were obtained from three different localities in the vicinity of Ann Arbor, Michigan. The fourth species Blepharisma sp. (BL), was obtained from a biological supply house. The Varieties are unknown except for PA which is Variety 3.

Culture techniques are those of Sonneborn (1950), with various modifications after Hairston and Kellerman (1965). Cultures were maintained at $15^{\circ} \mathrm{C}$ and all experiments were done at $25^{\circ} \mathrm{C}$. All cultures and experiments were kept in darkness-i.e., PB was without effective symbiotic algae.

Experiments were done in $10-\mathrm{ml}$ test tubes, filled with $5 \mathrm{ml}$ of culture. Each day a sample of $0.5 \mathrm{ml}$ was rapidly removed with a coarse pipette after the test tube had been vigorously shaken. The tubes were shaken after a section of rubber glove had been sterilized in hot distilled water and positioned as a covering for the test tube. Verification of the test tube sampling procedure was accomplished by repeated sampling and subsequent replacement of samples in several groups of test tubes. The sampling procedure was extensively experimented with to obtain a reasonably close correspondence between the mean and variance of a set of repeated samples; i.e., to assure a random sampling technique.

Bacterized culture medium was added to each culture to replace exactly the $0.5 \mathrm{ml}$ which had been removed as a sample. The culture medium had been aged for various lengths of time and was used in an orderly sequence based on this age. This was originally done to introduce a forced environmental oscillation on the experiments. Since absolutely no relationship between food age (food quality) and population growth or competition was observed anywhere in the study, the food source is considered as a constant in this paper.

\section{Population Growth}

The mathematical model used for the simple population growth experiments was the familiar logistic equation, $\mathrm{d} N / \mathrm{d} t=(r N / K)(K-N)$, where $N$ is the number of individuals, $K$ is saturation population density, and $r$ is the intrinsic rate of natural increase. The parameters $K, r$, and $N_{0}$ were estimated exactly as in Gause (1934) excepting the case of $\mathrm{PC}$ in which several points were discarded as wild points for purposes of estimating the parameters-not in plotting the 
figures. In all cases the intercept of the equation $\ln [(K-N) / N]=a+r t$ was used to estimate $N_{o}$, which was needed to generate the expected curve. Expected curves were obtained from the recurrence relation given by Leslie (1957),

$$
N_{t+1}=\lambda N_{t} /\left(1-b N_{t}\right)
$$

where $\lambda=\mathrm{e}^{r}$ and $b=(\lambda-1) / K$.

Statistical testing of the fit of the data to the model is at best difficult (Smith 1952). Leslie's (1957) procedure depends on the assumption of independence of observations from one time to the next. It was obvious by inspection that there was a rather high correlation between times within replicates-i.e., that replicate which had the highest value at time $t$ was most likely to be the replicate with the highest value at time $t+1$. Because of this statistical difficulty, and the difficulties raised by Smith (1952), the statistical testing of Leslie (1957) was not used.

Figure 1 presents the expected and observed values for population growth of $\mathrm{BL}$. The fit is

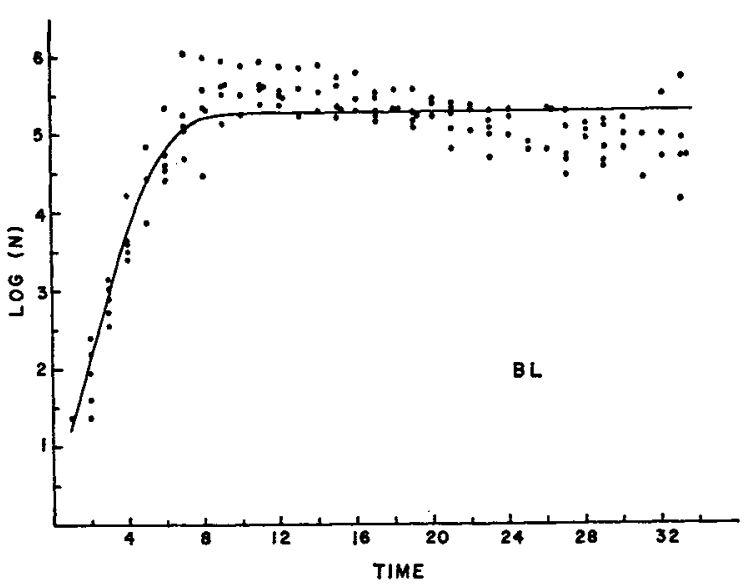

FIG. 1. Population growth of BL.

generally good, except that a systematic deviation of observed from expected is notable. This deviation is biased above the curve at early times, and below the curve at later times. The pattern is reminiscent of that obtained when time lags are added to the logistic equation (Cunningham 1954).

Using the equation of Wangersky and Cunningham (1956), the value of the reproductive time lag $\tau_{1}$, and density time lag $\tau_{2}$ were estimated. Systematic variation in the value of these two parameters provided no improvement of fit. If the deviations of fit are due to a time delay, the relationship is more complicated than that described by Wangersky and Cunningham's equation.

In Figure 2 are shown expected and observed values for $P B$. Of the four species this is the

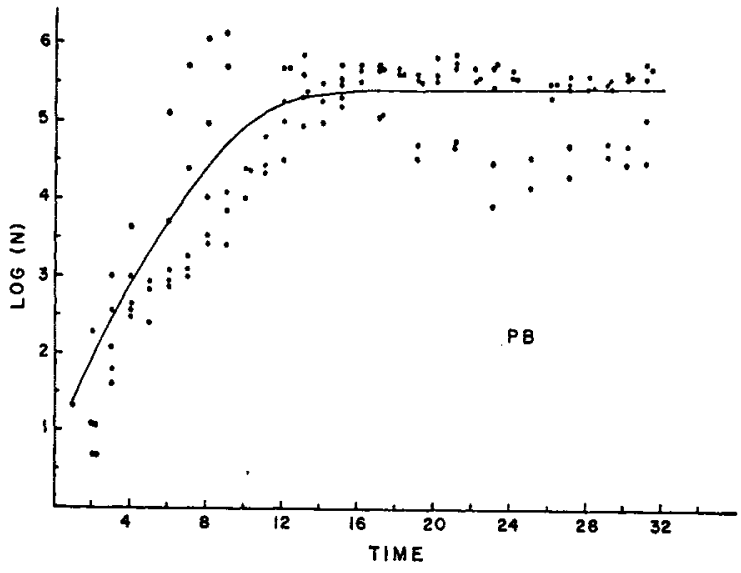

FIG. 2. Population growth of PB.

most highly variable in its population growth. The relatively large variance in observed values is understood by noting that two of the replicates are suggestive of a very large time lag and three are suggestive of a smaller, if at all existent, time lag. The curve predicted by the logistic (Fig. 2) seems to be an adequate representation of the basic trend of population growth in this species.

The expected and observed values for $\mathrm{PC}$ and $P A$ are shown in Figures 3 and 4 . The fits are obviously excellent and need no further discussion here.

The parameters for population growth for all four species are summarized in Table 1.

\section{Two-Way Competition}

The mathematical methods used here are even more inexact than those used in single species population growth. A smooth curve was drawn by eye through the observed data points, and estimates of $\alpha$ and $\beta$ were obtained for each time from 2 to 32, with the equations given by Gause (1934),

$$
\begin{aligned}
& \alpha=\frac{1}{N_{2}}\left[K_{1}-\frac{\left(\mathrm{d} N_{1} / \mathrm{d} t\right) K_{1}}{r_{1} N_{1}}-N_{1}\right] \\
& \beta=\frac{1}{N_{1}}\left[K_{2}-\frac{\left(\mathrm{d} N_{2} / \mathrm{d} t\right) K_{2}}{r_{2} N_{2}}-N_{2}\right]
\end{aligned}
$$

The $K$ 's and $r$ 's were obtained from the single species experiments. The $N$ 's were taken off of the smooth curve, and the derivatives were estimated as

$$
\frac{\mathrm{d} N_{t}}{\mathrm{~d} t}=\frac{N_{t+1}-N_{t-1}}{2}
$$

The resultant values of $\alpha$ and $\beta-31$ values of each for each of the six competition experimentswere highly variable within experiments. The values were exceptionally extreme at very early times and very late times. Intermediate values 


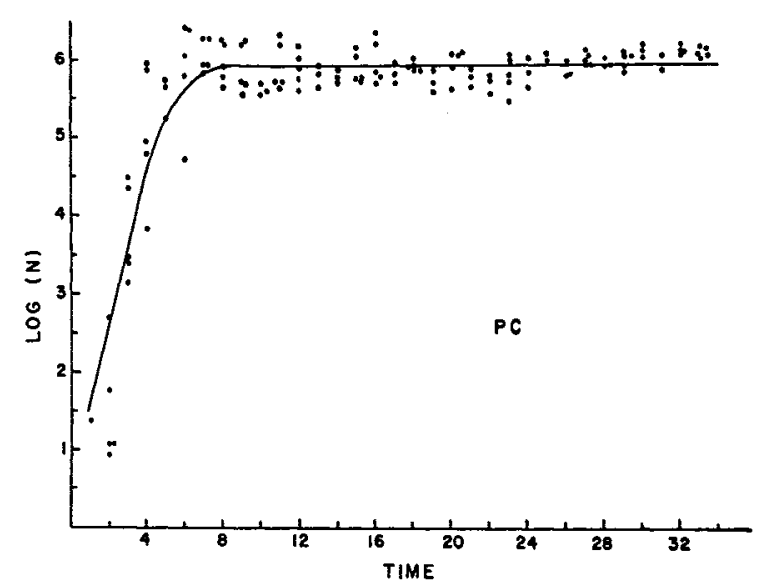

FIG. 3. Population growth of PC.

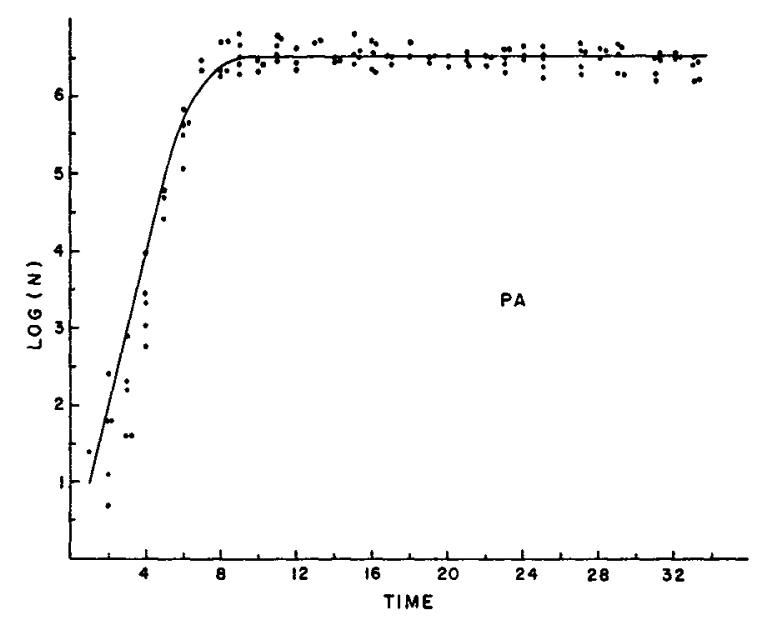

Fig. 4. Population growth of PA.

TABLE 1. Parameters of the logistic equation

\begin{tabular}{|c|c|c|c|}
\hline Species & $r$ & $K$ & $N_{0}$ \\
\hline $\begin{array}{l}\text { PA } \ldots \ldots \\
\text { PC...... } \\
\text { PB. } \ldots . \\
\text { BL } \ldots . .\end{array}$ & $\begin{array}{l}1.05 \\
1.07 \\
0.47 \\
0.91\end{array}$ & $\begin{array}{l}671 \\
366 \\
230 \\
194\end{array}$ & $\begin{array}{l}2.5 \\
5.0 \\
5.0 \\
3.0\end{array}$ \\
\hline
\end{tabular}

were somewhat less variable, but the variation seemed always to form some sort of trend. However, under the hypothesis of this paper, $\alpha$ and $\beta$ are defined as constants so the objective is to obtain those values of $\alpha$ and $\beta$ which produce the curve which best fits the observed data.

To obtain a reasonable first approximation to $\alpha$ and $\beta I$ took the average of the intermediate values as described above-i.e., the average of those values that tended to form a reasonably invariant clump. With these values, integral curves were computed using the predictor corrector method of Hamming (Ralston and Wilf 1960; see also System/360 Scientific Subroutine Pack- age 1968). Usually, these first approximations gave curves that rather poorly represented the observed data. A trial and error procedure was then pursued in which $\alpha$ and $\beta$ were systematically varied and the behavior of the integral curves investigated under this variation. It eventually became apparent that the curves obtained were about the best obtainable under the present model. In all cases the boundary conditions were considered as random variables, and were thus relatively free to vary in the trial and error procedure.

The comments about statistical testing made in the previous section apply equally well here. However, a relative measure of the goodness of fit is needed for a later section, and is introduced here to facilitate easy comparison of the goodness of fit of one experiment to that of another. The
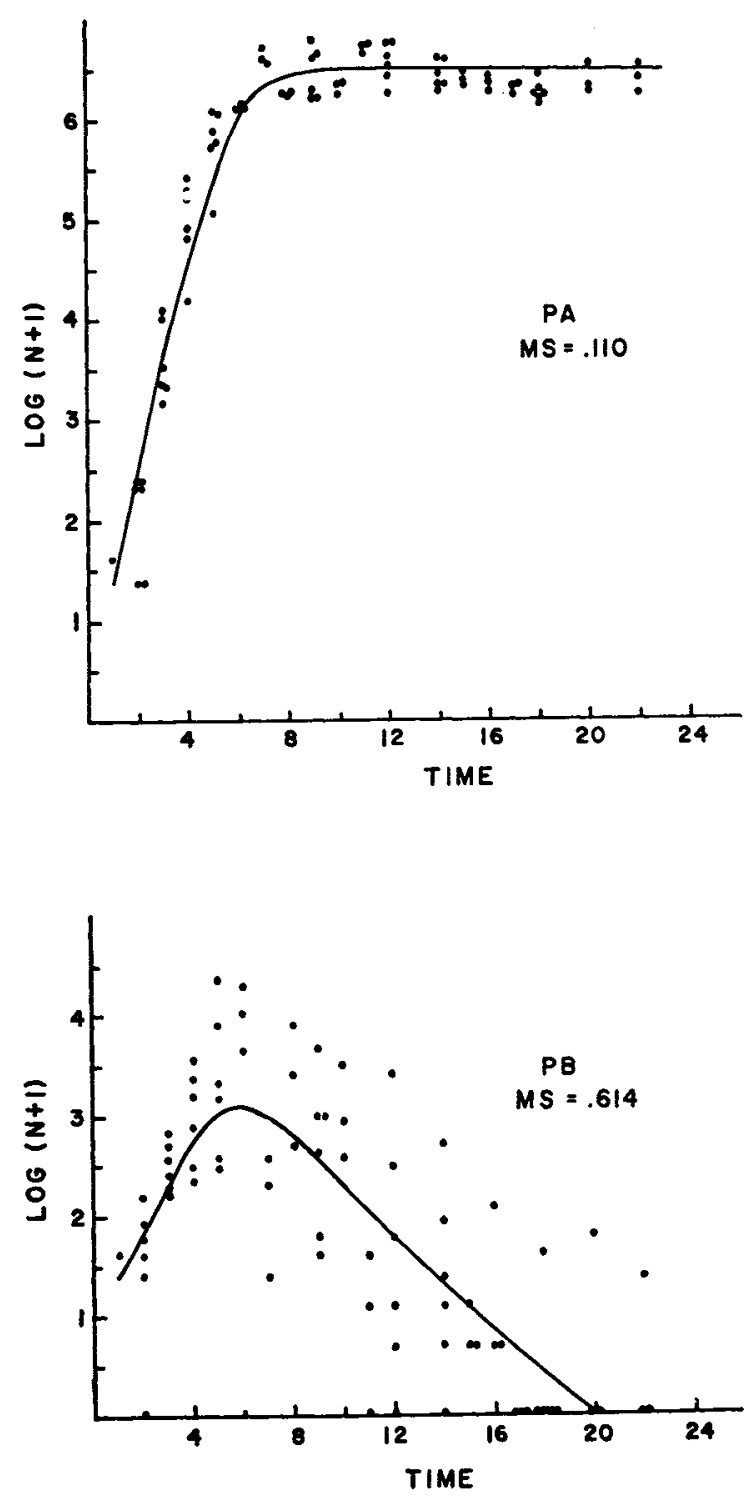

FIG. 5. Results of competition between PA and PB. 
arbitrary criterion used is mean square deviation of logs of observed from log of expected.

The results of the six pairwise competition experiments are presented in Figures 5-10, in order of decreasing mean square deviation. The aforementioned variability of $\mathrm{PB}$ is reflected in Figure 5 , but appears to be somewhat dampened by the presence of BL (Fig. 9). Figures 6 and 7 both show some indication of a time lag, but since the population growth data for these two species (PA and $P C$ ) alone did not suggest any frictional components, one must presume that the presence of a competitor is the factor which initiates a nonlinear response. However, the difference between the linear and nonlinear response in this case appears to be negligible.
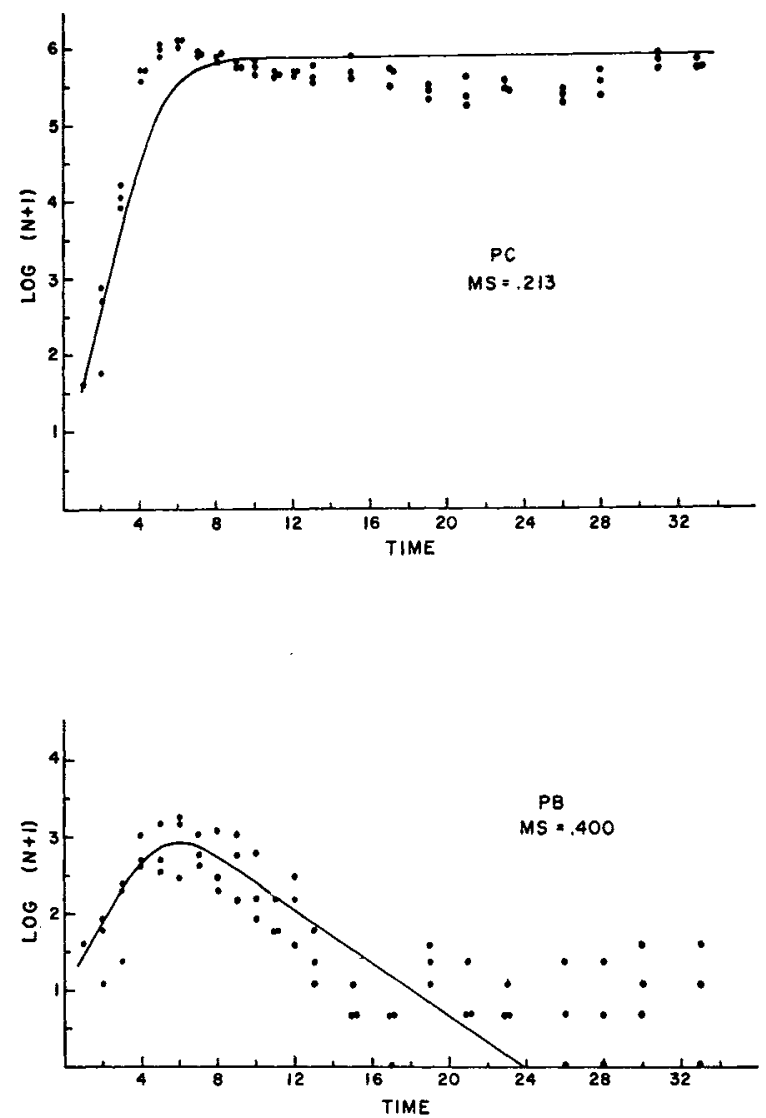

FIG. 6. Results of competition between $\mathrm{PC}$ and $\mathrm{PB}$.

Thus, the six cases of pairwise competition are represented quite well by the basic theory, minor deviations being interpretable as a result of unknown but insignificant frictional components or excessive inherent variability. Since at least the general trend of the data is in agreement with that of the model, these small deviations most likely will not interfere with the basic hypothesis to be tested.
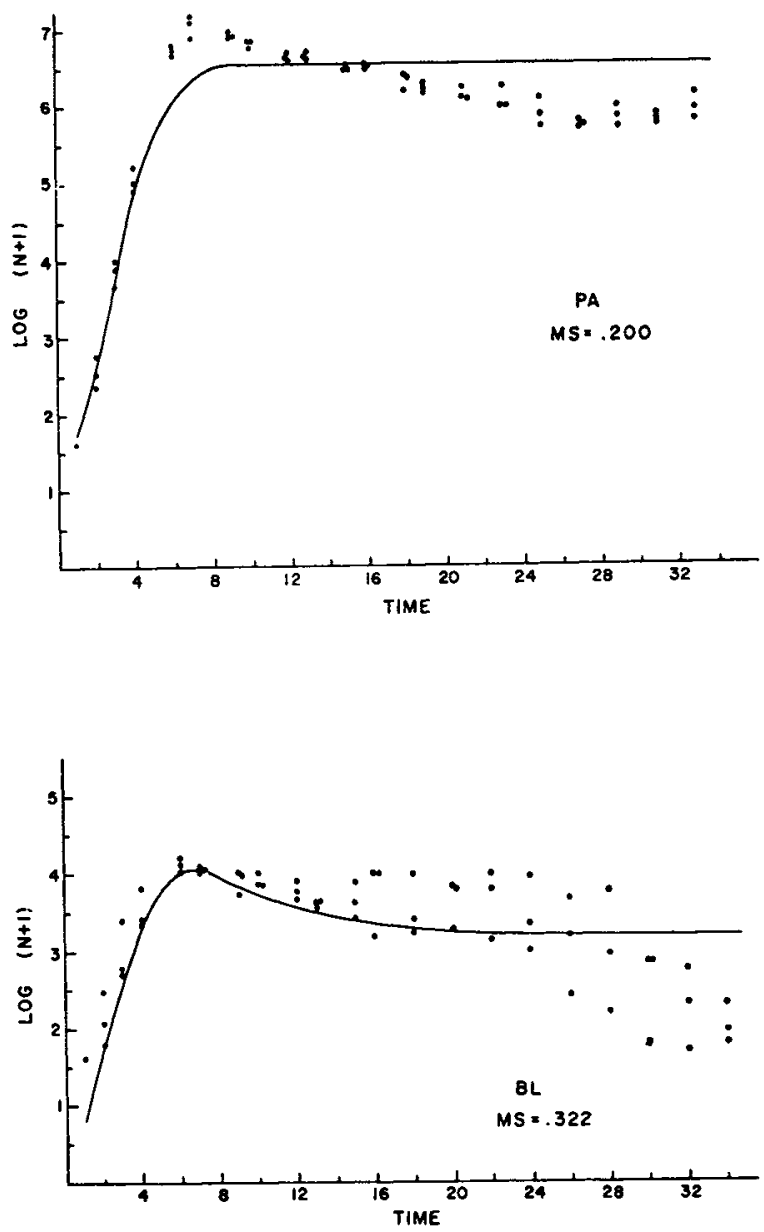

FIG. 7. Results of competition between PA and BL.

In Table 2 is presented the community matrix for this artificial community. The $\alpha_{i j}$ th entry refers to the effect of the $j$ th species on the $i$ th species.

\section{Four-Way Competition}

Having estimates of the intrinsic rate of natural increase and saturation density for each species, and estimates of all interaction coefficients on a pairwise basis, it is now necessary to substitute into equations (1) - four-way competition without higher order interactions-solve the equations, and see if the solution agrees with the data obtained from the laboratory experiments. It must be emphasized that up to this point all curves in this paper have been fit. That is, the expected curves are based on the data which they are describing and therefore cannot deviate too greatly. In the case of four-way competition, however, the expected curves are based on independent estimates of the parameters, estimates which were made from different experiments. As a consequence a reasonable expectation, if higher order 
TABLE 2. Community matrix for four species of protozoa in test tubes

\begin{tabular}{|c|c|c|c|c|}
\hline \multirow[b]{2}{*}{ ith Species } & \multicolumn{4}{|c|}{$j$ th Species } \\
\hline & PA & PC & PB & BL \\
\hline 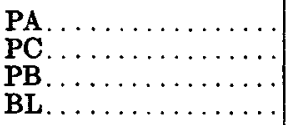 & $\begin{array}{l}1.00 \\
0.30 \\
0.50 \\
0.25\end{array}$ & $\begin{array}{l}1.75 \\
1.00 \\
0.85 \\
0.60\end{array}$ & $\begin{array}{r}-2.00 \\
0.50 \\
1.00 \\
-0.50\end{array}$ & $\begin{array}{r}-0.65 \\
0.60 \\
0.50 \\
1.00\end{array}$ \\
\hline
\end{tabular}

interactions are not important, is that the fit to the four-way situation is, at best, as good as the fits to the pair-wise competitions. Though it cannot be expected that the four-way competition experiments will fit the model as well as the twoway competition, it is not clear exactly how "good" a fit is necessary to judge a model as being valid. Such a judgment, of course, depends on the purposes for which the model is to be used. In the present case it seems that there are three vaguely distinquishable levels of purpose which might be used in helping to judge the validity of this model. First, if only the equilibrium conditions of the model are needed-say in predicting the maximum number of species in an equilibrium communitywe might only require that the model predict accurately which species will persist and which will
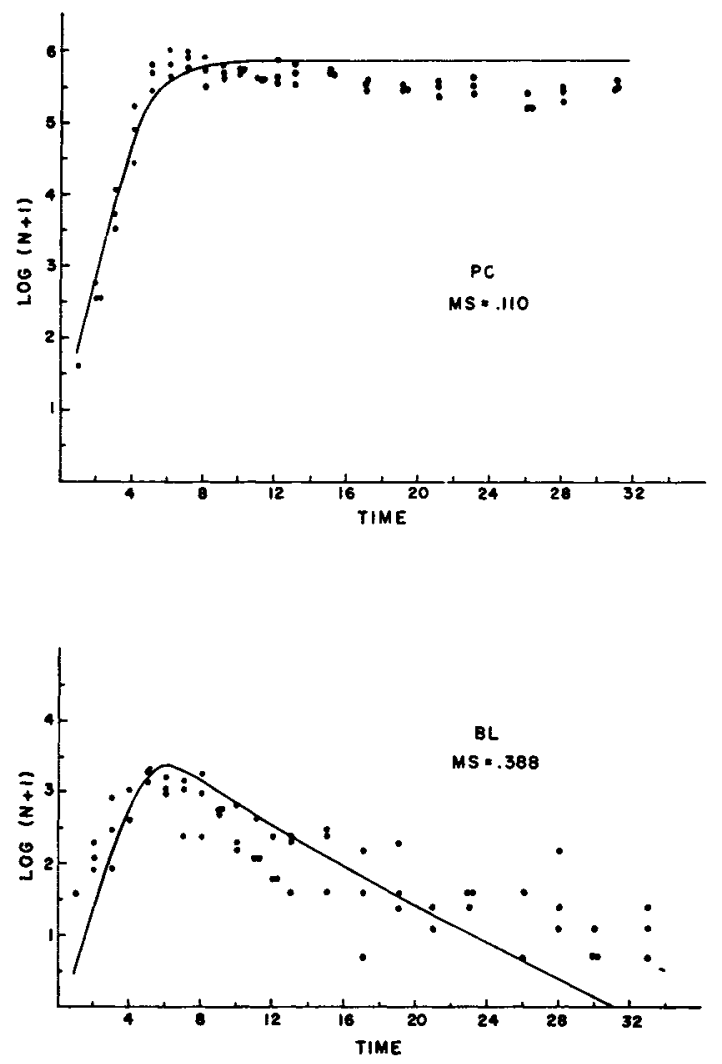

FIG. 8. Results of competition between PC and BL.
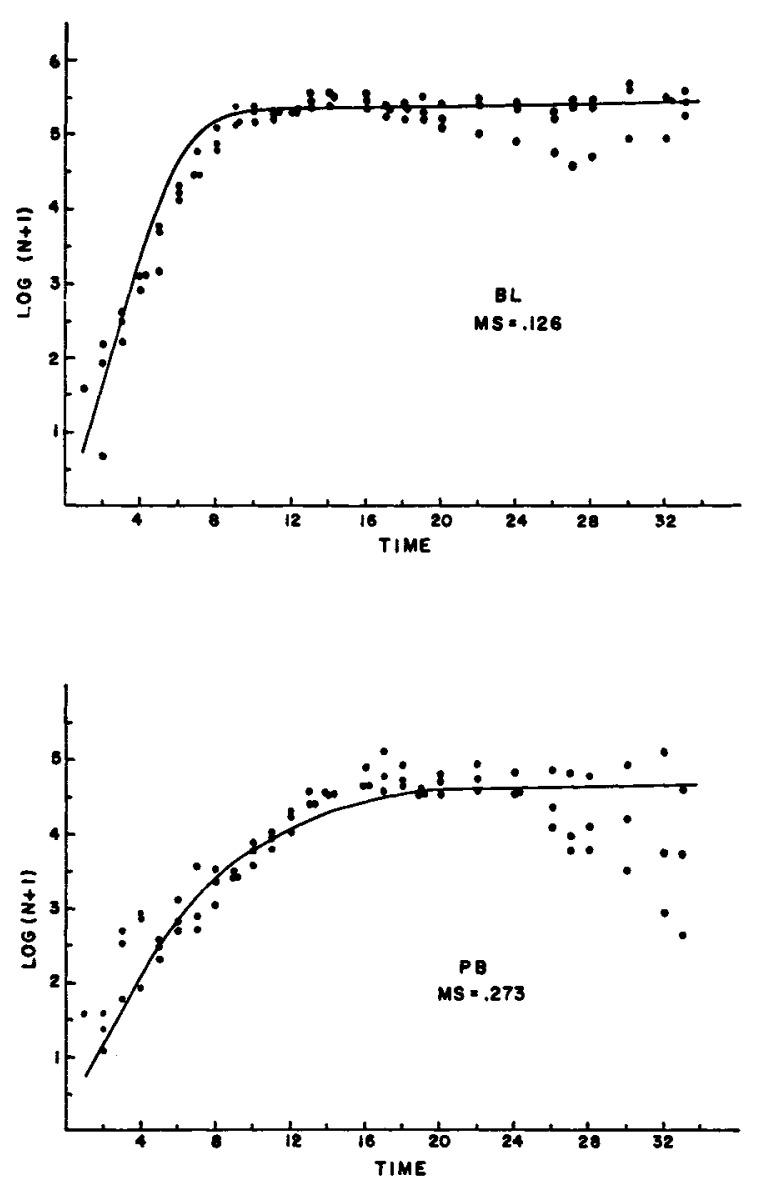

FIG. 9. Results of competition between $\mathrm{BL}$ and $\mathrm{PB}$.

go to extinction. Second, the relative numbers of individuals at any particular point in time may be desired-say, in a theoretical study of the relative abundance of species-or, more or less equivalently, the general behavior of the populations within the framework of the larger system must be known-i.e., does species $i$ oscillate, does it tend to be peaked and then drop off fast, and similar general questions. Under this requirement, we accept the model as valid if the general shape of the curves reflects the general trend of the data. Third, we may wish to make very precise predictions with the model such as exactly how much pesticide must be applied at time $x$ to produce $y$ per cent change in species $i$ by time $x+a$. At this level we require the fit to fourway competition to be almost as good as the fit to the two-way competitions. Certainly these three levels are not discrete, but are merely useful points in a continuum, defined so as to be able to judge the relative validity of the model as applied to a simple community.

In Figures 11-14 are shown the results of simulating four-way competition with the community 

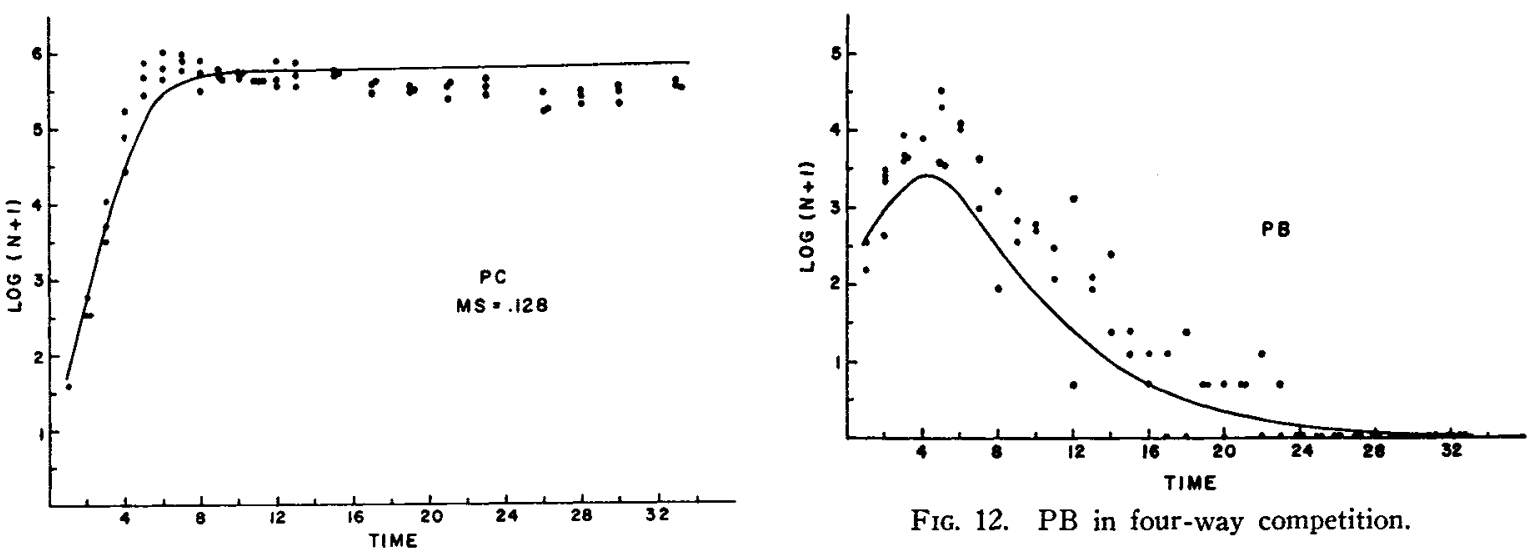

FIG. 12. $\mathrm{PB}$ in four-way competition.
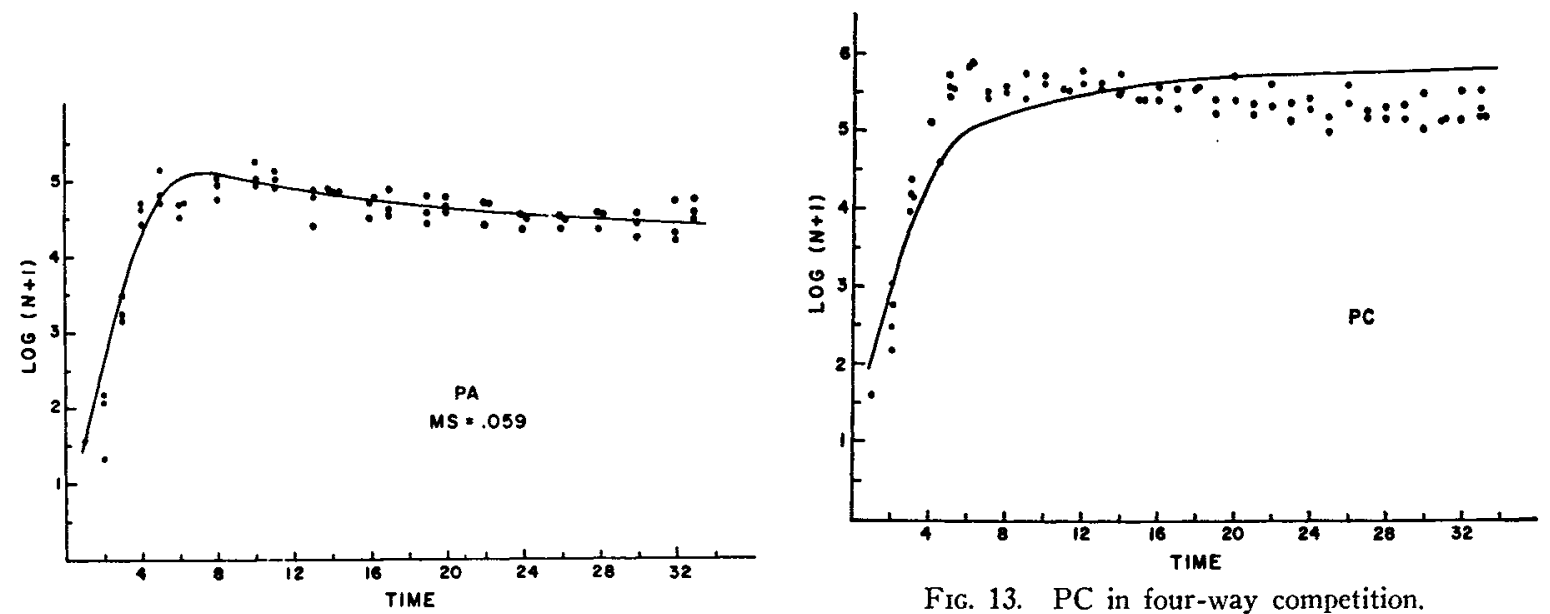

FIg. 10. Results of competition between PC and PA.

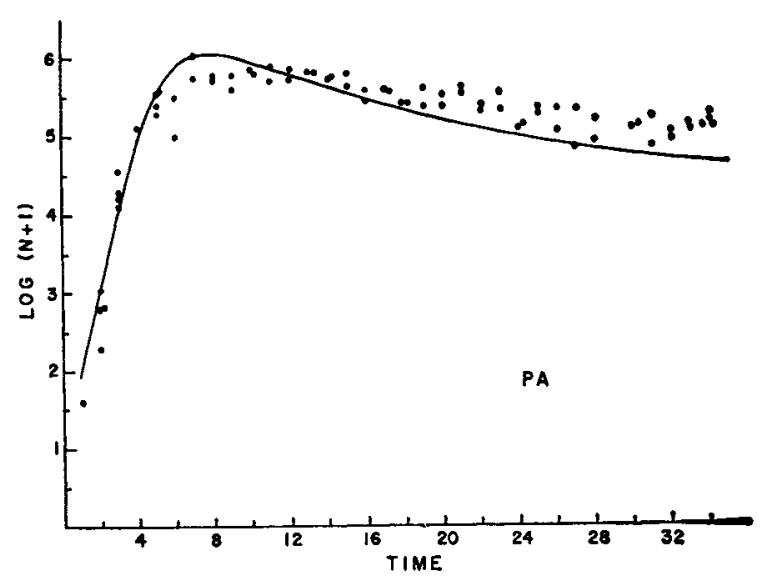

FIG. 11. PA in four-way competition.

matrix of the last section and the $r$ 's and $K$ 's from the single species population growth experiments. Also shown are the observed data from the fourway competition experiments.

Under the first criterion of validation-only the final outcome is desired-the data are described perfectly by the model. The model predicts that $\mathrm{PB}$ will be extinct; $\mathrm{BL}$ will be at a very low popu-

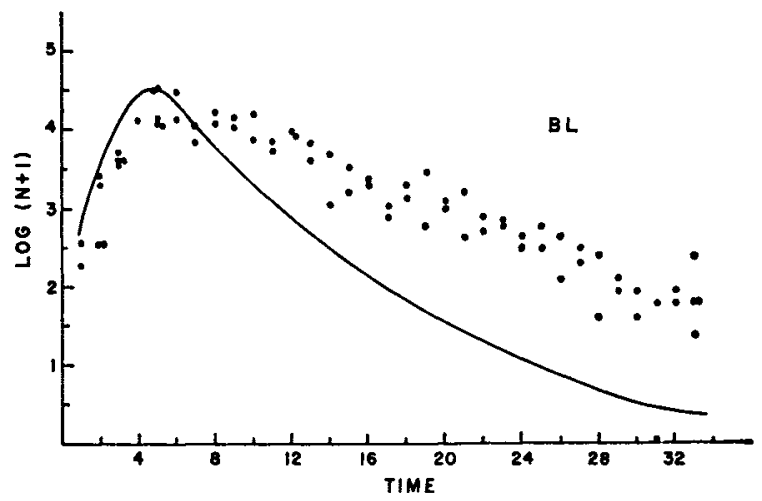

FIG. 14. BL in four-way competition.

lation density; PA and $\mathrm{PC}$ will have higher densities. As can be easily seen from the figures, the data correspond exactly to these predictions.

Likewise under the second criterion the model is validated. The general trends are predicted in every case, and the rank order of abundance is as predicted, at virtually all points in time. The general trends of growth, peaking, and decay are predicted fairly accurately. The early peak for $\mathrm{PC}$ reflected in the experiments is essentially the 
same as that species exhibits in two-way competion (see Fig. 6, 8, and 10).

Finally, consider the third level of validation. Somewhere in between the second and third level the validation becomes questionable. The third level requires that precise predictions be made. The three species PC, PA and PB conform quite well, but BL seems to deviate excessively from the expected. However, as discussed previously, it is not sufficient to examine the data and expecttation alone, but they must be considered in the light of the pair-wise experiments, since the correspondence between expected and observed can only be as good as that of the basic model. It is for this reason that the mean square deviations were computed earlier (Fig. 5-10). In Table 3 are listed the pooled mean squares for each species-pooled over all two-way competitions in which that species was a part-and the mean

TABLE 3. Mean squares for two-way and four-way competition experiments, and ratio between the two (taking largest mean square as numerator). Numbers in italics are degrees of freedom

\begin{tabular}{|c|c|c|c|c|}
\hline \multirow[b]{2}{*}{ Statistic } & \multicolumn{4}{|c|}{ Species } \\
\hline & PA & $\mathrm{PC}$ & PB & BL \\
\hline $\begin{array}{l}\text { Pooled mean } \\
\text { square for } 2 \text {-way } \\
\text { competition } \\
\text { Mean square for } \\
\text { 4-way competi- } \\
\text { tion } \\
\text { Ratio }\end{array}$ & $\begin{array}{l}0.11212 \\
0.1074 \\
1.1\end{array}$ & $\begin{array}{ll}0.15 & 197 \\
0.22 & 74 \\
1.5 & \end{array}$ & $\begin{array}{l}0.43 \quad 230 \\
0.36 \quad 74 \\
1.2\end{array}$ & $\begin{array}{l}0.26 .209 \\
1.28 \quad 74 \\
4.9\end{array}$ \\
\hline
\end{tabular}

squares for the same species in four-way competition. The first three species show very similar mean squares between two-way and fourway competition; indeed $\mathrm{PA}$ and $\mathrm{PB}$ are even better predicted in the case of four-way than in the case of two-way competition. The case of $\mathrm{BL}$ is somewhat less encouraging. The mean square is roughly five times greater in four-way than in two-way. The fact that the experimental points are always greater than predicted, implies that whatever higher order interaction there may be, they act solely to decrease the competitive effect felt by BL. They are noncoalition type higher order interactions.

However, it might be argued that if one is interested in this model from the standpoint of the community, it is not really valid to compare predictions on a specific basis. We should instead be interested in the general performance of the model in predicting community dynamics. To this end the sum of squares pooled over all two-way competition cases was computed as 0.246 and the same pooled over all four-way cases was 0.487 , only a two-fold difference. Thus, the four-way model predicted about half as well as the two-way model, implying that, from the point of view of the community as a whole, the higher order interactions are rather unimportant.

The above discussion is similar to the problem of multiple comparisons tests in statistics. Given a group of means, we wish to test for differences. In setting an error rate we are faced with deciding on the relative importance of pair-wise or experiment-wise rates. That is, is it more important to minimize the probability of making any mistake at all, or the probability that a given pair of means may be judged different when they are truly the same. Similarly in the present context, we are faced with deciding whether we want the model to make predictions about the community as a whole, or about the components of the community. The former criterion provides a single judgment of the model. The latter provides as many judgments as there are components.

Thus, if we are concerned with judging the present model under the third criterion-absolutely precise predictions-and if we are interested in the individual components, the noninteractive model is "good" three times and "bad" once. On the other hand, it appears to be simply "good" on the level of the whole community.

\section{CONCLUSION}

The conclusions of these experiments were basically stated in the previous section. However, it might be well at this point to recall statements made earlier about the choice of the experimental system. It was the author's prejudice that higher order interactions are very important in nature. Thus, the experimental system was chosen to maximize the chance of not finding higher order interactions, hoping, of course, that even then such interactions would be significant. Then, because of the way the experimental system was put together, one could make a fairly powerful statement about the universal occurrence of higher order interactions in nature. Unfortunately, the basic models proved to be excellent predictors (i.e., higher order interactions did not seem important), and one can only cite this study as a single example of the insignificance of higher order interactions.

\section{ACKNOWLEDGMENTS}

I am deeply indebted to Nelson G. Hairston who provided guidance and encouragement throughout the course of this work. I wish to thank James T. MacFadden, Lawrence B. Slobodkin, and Donald W. Tinkle for constructive comments and criticisms. I also am thankful to Douglas J. Futuyma for much valuable advice, and to 
my wife Jean for assistance in tabulating data and typing the manuscript. Computer time was granted by the University of Michigan Computation Center. This research was supported by an NSF summer fellowship for graduate teaching assistants, and an $\mathrm{NIH}$ predoctoral fellowship.

\section{Literature Cited}

Cunningham, W. J. 1954. A nonlinear differentialdifference equation of growth. Proc. Nat. Acad. Sci. 40: 708-713.

Ehrlich, P. R. and L. C. Birch. 1967. The "balance of nature" and "population control." Amer. Naturalist 101: 97-107.

Fisher, R. A., A. S. Corbet, and C. B. Williams. 1943. The relation between the number of species and the number of individuals in a random sample from an animal population. J. Aninal Ecol. 12: 42-58.

Garfinkel, D. 1967. A simulation study of the effect on simple ecological systems of making rate of increase of population density-dependent. J. Theoret. Biol. 14: 46-58.

Gause, G. F. 1934. The struggle for existence. Williams and Wilkins, Baltimore.

Hairston, N. G. 1959. Species abundance and community organization. Ecology 40: 404-416.

Hairston, N. G. and G. W. Byers. 1954. The soil arthropods of a field in southern Michigan. A study in community ecology. Contrib. Lab. Vert. Biol., Univ. Mich. 64: 1-37.

Hairston, N. G., F. E. Smith, and L. B. Slobodkin. 1960. Community structure, population control, and competition. Amer. Naturalist 94: 421-425.

Hairston, N. G. and S. L. Kellermann. 1965. Competition between varieties 2 and 3 of Paramecium aurelia: the influence of temperature in a food limited system. Ecology 46: 134-139.

Hairston, N. G., J. D. Allan, R. K. Colwell, D. J. Futuyma, J. Howell, J. D. Mathias, and J. H. Vandermeer. 1969. The relationship between species diversity and stability: an experimental approach with protozoa and bacteria. Ecology 49: 1091-1101.

International Business Machines, 1968; System/360 scientific subroutine package (360A-CM-03X) Version III Programmer's manual. IBM Technical Publications Department, White Plains, N. Y.

Kendall, D. G. 1948 . On some modes of population growth leading to R. A. Fisher's logarithmic series distribution. Biometrika 35: 6-15.

Leslie, P. H. 1957. An analysis of the data for some experiments carried out by Gause with populations of the Protozoa, Paramecium aurelia and Paramecium caudatum. Biometrika 44: 314-327.

Levins, R. 1968. Evolution in changing environments. Some theoretical explorations. Monographs in population biology, Princeton Univ. Press.

MacArthur, R. H. 1955. Fluctuations of animal populations, and a measure of community stability. Ecology 36: $533-536$.

- 1960. On the relative abundance of species. Amer. Naturalist 94: 25-36.

MacArthur, R. H. and R. Levins. 1967. The limiting similarity, convergence, and divergence of coexisting species. Amer. Naturalist 101: 377-385.

Margalef, R. 1957. Information theory in ecology. (English trans. by Hall, W.) Gen. Systems 3: 3671.

Murdoch, W. W. 1966. Community structure, population control, and competition-a critique. Amer. Naturalist 100: 219-226.

Pielou, E. C. 1966a. Species-diversity and patterndiversity in the study of ecological succession. J. Theor. Biol. 10: 370-383.

- 1966b. The measurement of diversity in different types of biological collections. J. Theor. Biol. 13: $131-144$.

Preston, F. W. 1948. The commonness, and rarity, of species. Ecology 29: 254-283.

- 1962a. The canonical distribution of commonness and rarity. Ecology 43: 185-215.

- $1962 \mathrm{~b}$. The canonical distribution of commonness and rarity. Part II. Ecology 43: 410-432.

Ralston, A. and H. S. Wilf. 1960. MathematicaI methods for digital computers. Wiley, New York.

Slobodkin, I. B., F. E. Smith, and N. G. Hairston. 1967. Regulation in terrestrial ecosystems, and the implied balance of nature. Amer. Naturalist 101: $109-124$.

Smith, F. E. 1952. Experimental methods in population dynamics: a critique. Ecology 33: 441-450.

Sonneborn, T. M. 1950. Methods in the general biology and genetics of Paramecium aurelia. J. Exper. Zool, 113: 87-147.

Vandermeer, J. H. 1968. The structure of communities as determined by competitive interactions: $a$ theoretical and experimental approach. Ph.D. thesis, Univ. Mich., Ann Arbor.

Wangersky, P. J. and W. J. Cunningham. 1956. On time lags in equations of growth. Proc. Nat. Acad. Sci. Wash. 42: 699-702.

Watt, K. E. F. 1964. Comments on fluctuations of animal populations and measures of community stability. Can. Entomol. 96: 1434-1442.

\title{
LIGHT CONTROL OF AQUATIC INSECT ACTIVITY AND DRIFT
}

\author{
JoHN E. Bishop ${ }^{1}$ \\ Department of Biology, University of Waterloo, Waterloo, Ontario, Canada
}

(Received November 27, 1968; accepted for publication February 4, 1969)

\footnotetext{
Abstract. Investigations on aquatic insect activity, measured as drift in an artificial stream system with rigid light and temperature conditions, demonstrated a light-controlled, labile, exogenously-mediated activity rhythm. A threshold value for light, incident at the water surface, which when decreased led to high drift rates, and when increased suppressed activity,

'Present address: School of Biological Sciences, University of Malaya, Kuala Lampur, Malaysia.
} 